\title{
A Study on Pulmonary Function of Adolescent Bengalee Trainee Bharatnatyam Dancers
}

\author{
Neepa Banerjee $^{1}$, Tanaya Santra ${ }^{1}$, Sandipan Chaterjee ${ }^{1}$, Ayan Chatterjee $^{1}$, Surjani Chatterjee ${ }^{1}$, Ushri Banerjee $^{2}$, \\ Shankarashis Mukherjee ${ }^{1}$, Indranil Manna ${ }^{3, *}$ \\ ${ }^{1}$ Human Performance Analytics and Facilitation Unit, Department of Physiology, \\ ${ }^{2}$ Department of Applied Psychology, University Colleges of Science and Technology, University of Calcutta, 92 APC Road, Kolkata, \\ W.B., India. \\ ${ }^{3}$ Department of Physiology, Midnapore College, Midnapore, W. B., India, \\ *Corresponding author: indranil_manna@yahoo.com
}

Received June 17, 2014; Revised July 28, 2014; Accepted September 25, 2014

\begin{abstract}
Dance, a type of art that generally refers to the rhythmic body movement, is performed in many different cultures. It provides an energetic, non-competitive form of exercise which has potential positive impacts on physical health and may enhance fitness level. On the other hand, pulmonary function test is a non invasive and simple technique used for the assessing lung function status. Practicing dancing as a physical activity may have some impact on lung function variables. In this backdrop a study was conducted to assess the effect of dancing exercise on the pulmonary function indices in terms of VC, $\mathrm{FEV}_{1}, \mathrm{FEV}_{1} \%$, PEFR in the 31 adolescent Bengalee females receiving training in Bharatnatyam dancing. It has been found that individuals receiving dancing training have significant $(\mathrm{P}<0.05)$ favorable impact on pulmonary status compared to control group consisting of 39 individuals of same age and socio economic status.
\end{abstract}

Keywords: physical activity, respiratory status, spirometry, physical fitness, adolescent

Cite This Article: Neepa Banerjee, Tanaya Santra, Sandipan Chaterjee, Ayan Chatterjee1, Surjani Chatterjee, Ushri Banerjee, Shankarashis Mukherjee, and Indranil Manna, "A Study on Pulmonary Function of Adolescent Bengalee Trainee Bharatnatyam Dancers." American Journal of Sports Science and Medicine, vol. 2, no. 5A (2014): 45-47. doi: 10.12691/ajssm-2-5A-10.

\section{Introduction}

Dance is a type of physical activity accompanied with music of a certain tempo, rhythm and dynamics. It is performed in many different cultures and can make a significant contribution to the healthy-living agenda [1]. It also reduces stress, increases stamina [2] and helps in calorie expenditure and results in weight loss [3]. The likelihood of suffering from many diseases like cardiovascular diseases, Parkinson's diseases, obesity, and diabetes get reduced on regular practicing of dance [2]. It involves continuous body movements and thus may have some impact on pulmonary function parameters, as well. The test to assess pulmonary function, Pulmonary Function Test is a non invasive and useful tool not only for to assess pulmonary function and but also to detect any airway obstruction and other air tapping related disorders [4]. These simple technique uses inter alia provides the information about the degree of severity of the pulmonary disorders. It has been reported that physical exercises improve the respiratory status as well as physical fitness. But information regarding the impact of dancing on respiratory status particularly in Bengalee female adolescents is not much available. In this backdrop, a study has been undertaken to assess the impact of dancing on lung function parameters in adolescent Bengalee females.

\section{Materials and Methods}

At first, institutions imparting training on dancing were approached for obtaining permission to work on the individuals receiving Bharatnatyam dance training. On obtaining initial consent, the names of volunteers were enlisted and the procedural requirement was explained to them elaborately. The study was conducted on randomly chosen female adolescents, aged between 14-17 years, generally living in and around Kolkata, the capital of Indian province West Bengal. 31 female individuals receiving training in Bharatnatyam dance for a minimum period of 3 years and practising it regularly for at least half an hour for 6 days a week, constituted the dancing group (DG) and 39 female individuals of comparable age and socio- economic background, but not receiving training in any form of dance or exercise, constituted the control group (CG). Individuals having any history of personal or familial (self-reported) lung problem were excluded. The study was carried out on mutually convenient dates and the required measurements were taken in the morning hours. The age (years) and information about duration of different daily activities 
were recorded in pre-designed schedules. Basic anthropometric parameters like body height (to the nearest accuracy of $0.1 \mathrm{~cm}$ ) using an anthropometric rod, and body weight (to the nearest accuracy of $0.1 \mathrm{~kg}$ ) using a pre calibrated weighing scale, with subjects in light clothing and without shoes, were measured and BMI was calculated [5]. After these initial recordings, the subjects were asked to take rest for at least a period of 15 minutes, pulmonary function test was carried out subsequent to the subjects being familiarized with the study protocol [6], using Jaeger Flowscreen pro [7]. Pulmonary function variables like vital capacity (VC), forced expiratory volume in $1^{\text {st }}$ second $\left(\mathrm{FEV}_{1}\right)$ were measured and $\mathrm{FEV}_{1} \%$ values were obtained. Peak expiratory flow rate (PEFR) was also measured using portable peak flow meter and the obtained values were expressed in BTPS [8]. The obtained data were subjected to test of significance to find out any significant difference. $P$ value lower than $0.05(P<0.05)$ was considered significant.

\section{Results}

The basic physical profile of both dancing and control group in terms of age (years), body height $(\mathrm{cm})$, body weight (kg) and Body Mass Index have been presented in the Table 1 .

Table 1. Demographic Profile of the study participants

\begin{tabular}{|c|c|c|}
\hline Parameters & DG & CG \\
\hline Age (years) & $15.5 \pm 1.06$ & $15.3 \pm 0.73$ \\
\hline Body Height $(\mathrm{cm})$ & $150.4 \pm 4.84$ & $157.1 \pm 5.77$ \\
\hline Body Weight $(\mathrm{kg})$ & $44.6 \pm 1.95$ & $57.5 \pm 3.85$ \\
\hline Body Mass Index (kg.m $\left.{ }^{-2}\right)$ & $19.9 \pm 1.60$ & $23.3 \pm 1.47$ \\
\hline
\end{tabular}

Values are in mean $\pm \mathrm{SD}$; DG= dancing group, $\mathrm{CG}=$ control group

Comparison between DG and CG in respect of pulmonary function parameters VC (l), $\operatorname{FEV}_{1}\left(\operatorname{lomin}^{-1}\right)$, $\mathrm{FEV}_{1} \%$ and PEFR $\left(\mathrm{l}_{\mathrm{min}}^{-1}\right)$ have been graphically presented in Figure 1.

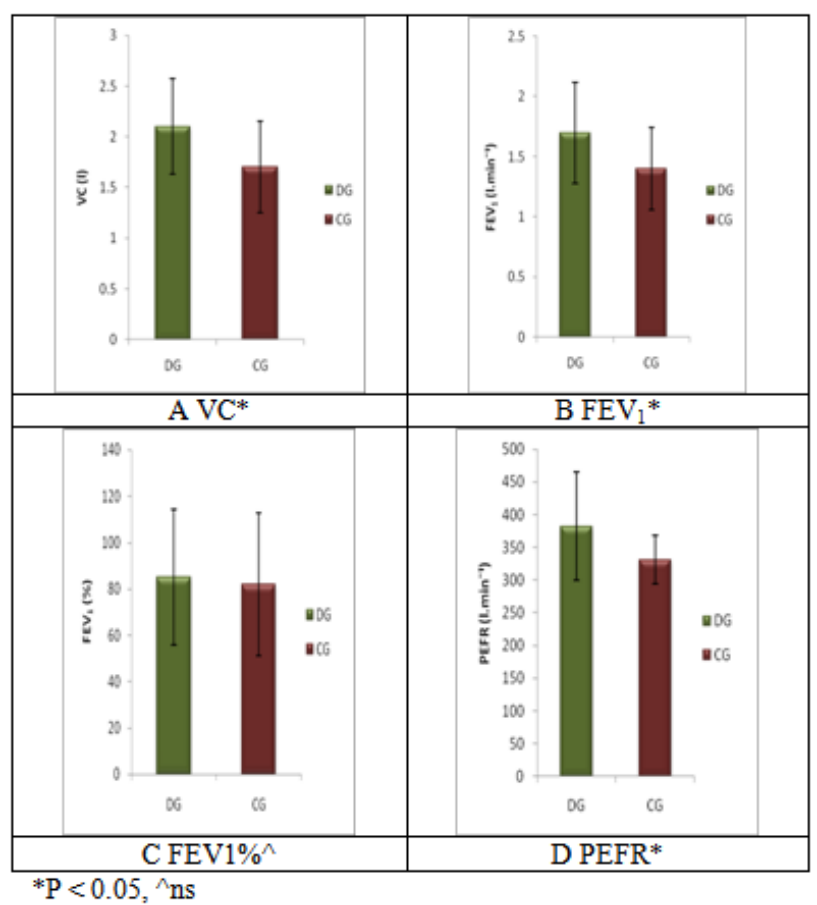

Figure 1. Comparison between DG and CG individuals in respect of pulmonary function parameters

\section{Discussion}

Sedentary lifestyle leads to lower level of cardiorespiratory fitness and is recognised as an important cause of morbidity and mortality [9]. On the other hand, exercise is considered an important component of pulmonary rehabilitation for patients with chronic obstructive pulmonary disease (COPD) [10]. In the present cross sectional study, the relation between Bharatnatyam dance as a physical activity and cardio-respiratory function for healthy female adolescents aged 14 - 17 years was observed.

As shown in the Table 1, the DG and CG individuals do not differ significantly in terms of age. DG individuals have been found to have lower BMI values compared to their CG counterparts.

But, there is significant difference $(\mathrm{P}<0.05)$ between DG and CG volunteers in respect of the pulmonary function variables VC, FEV 1 , PEFR (Figure 1).

The higher $\mathrm{VC}$ and $\mathrm{FEV}_{1}$ values of the dancing group indicates that physical activity like dancing may have a positive impact on lung functions. The findings are in agreement with the observations from the other study carried out on adolescent males and females depicting that as the exercise level increases, FVC, $\mathrm{FEV}_{1}$ value increases [11]. PEFR, an effort dependent value which can easily detect airway obstruction, is higher in DG study participants compared to their CG counterparts; this finding is in consonance with the observations of Chaitra et al [10] in which moderate intensity aerobic training of young Indian males improved pulmonary function variables FVC, $\mathrm{FEV}_{1}$, and PEFR but not $\mathrm{FEV}_{1} \%$. In the present study similar trend has been observed. The result of the present study is also in agreement with other studies conducted on individuals of sedentary lifestyle [10,12,13] in which the planned physical activity improved pulmonary function in healthy adults. The findings of the present study is also in consonance with the observations of Quin et al [1], where practicing creative dance on a regular basis increased lung capacity and also aerobic capacity in adolescent females.

\section{Conclusion}

In the light of the observations discussed, it may be concluded that receiving training in Bharatnatyam dance on a regular basis has favorable impact on pulmonary function status, as observed in the present study conducted on Bengalee adolescent trainee Bharatnatyam danseuses.

\section{Acknowledgements}

The authors of the paper are thankful to the volunteers for participating in the study, to the head of the institutions and to University of Calcutta.

\section{References}

[1] Quin E, Redding E, Frazer L, The effects of an eight-week creative dance programme on the physiological and psychological status of 11-14 year old adolescents: An experimental study, Hampshire Dance and Laban, 2007. 
[2] Bremer Z, Dance as an Exercise, The British Journal of General Practice, 57, 166, 2007.

[3] Callahan C, Dance as an Exercise to Lose Weight, (2010).

[4] IP MSM, Karlberg EM, Chan KN, Karlberg JPE, Luk KDK, Leong JCY, Lung function reference values in chinese children and adolescents in Hong Kong, Am J Respir Crit Care Med, 162, 430-435, 2000

[5] Mukherjee S, Banerjee N, Chatterjee S, Chatterjee S, Chatterjee A, Santra T, and Saha B, Effect of Bharatnatyam Dancing on Body Composion of Bengalee Female Children, American Journal of Sports Science and Medicine, 2 (1), 56-59, (2014).

[6] American Thoracic Society, Lung function testing: selection of reference values and interpretative strategies, Am Rev Respir Dis, 144, 1202-1218, 1991.

[7] Rodrigues MT, Fiterman-Molinari D, Barreto SSM, Fiterman J, The role of the $\mathrm{FEF}_{50 \%} / 0.5 \mathrm{FVC}$ ratio in the diagnosis of obstructive lung diseases, J Bras Pneumol, 36, 44-50, 2010.

[8] Miller MR, Hankinson J, Brusasco V, Burgos F, Casaburi R, Coates A, Crapo R, Enright P, Grinten CPM van der, Gustafsson P, Jensen R, Johnson DC, MacIntyre N, McKay R, Navajas D, Pedersen OF, Pellegrino R, Viegi $G$ and Wanger J, Standardisation of spirometry, Eur Respir J, 26, 319-338, 2005.
[9] Dunn AL, Marcus BH, Kampert JB, et al. Comparison of lifestyle and structured interventions to increase physical activity and cardiorespiratory fitness: a randomized trial. JAMA, 281, 327-34, 1999.

[10] Chaitra B, Narhare P, Puranik N, Maitri V, Moderate intensity aerobics training improves pulmonary function in young Indian men, Biomedical Research, 23, 231-233, 2012.

[11] Holmen TL, Barrett-Connor E, Clausen J, Holmen J, Bjermer L, Physical exercise, sports, and lung function in smoking versus nonsmoking adolescents, Eur Respir J, 19, 8-15, 2002.

[12] Cheng YJ, Macera CA, Addy CL, Sy FS, Wieland D, Blair SN, Effects of physical activity on exercise tests and respiratory function, Br. J. Sports Med, 37, 521-528, 2003.

[13] Mehrotra PK, Varma N, Tiwari S, et al, Pulmonary function in Indian sportsmen playing different sports, Indian $J$ Physiol Pharmacol, 42, 412-416, 1998. 